\title{
Gliomas in rodent whisker barrel cortex: a new tumor model
}

Eric W. Sherburn, M.D., John E. Wanebo, M.D., Paul Kim, B.S., Sheng-Kwei Song, Ph.D., Michael R. Chicoine, M.D., and Thomas A. Woolsey, M.D.

Departments of Neurological Surgery and Chemistry, Washington University School of Medicine, St. Louis, Missouri

Object. Surgical treatment of gliomas is difficult because they are invasive. Invasion of essential cortex often limits or precludes surgical resection. A tumor model was developed in which the rodent whisker barrel cortex was used to examine how gliomas affect cortical function and structure.

Methods. Both DBT (mouse) and C6 (rat) glioma cell lines were grown in culture and labeled with the fluorescent marker Dil in vitro. Labeled tumor cells were then injected into the whisker barrel cortex of adult mice and rats. Neurological assessments were made daily and magnetic resonance (MR) images were obtained. Animals were killed by perfusion 6 to 14 days after injection, and histological sections were prepared and studied.

Tumors were found in all 20 rats and 10 mice that had been injected with the C6 and DBT cell lines, respectively. The animal cells had been labeled with Dil in vitro, and all in vivo tumors proved to be Dil positive. The MR images revealed the tumor locations and serial MR images demonstrated tumor growth. Histological evaluation confirmed the location of the tumor and the disruption of barrel cortex architecture.

Conclusions. Both DBT and C6 glioma cell lines can be used to generate malignant glial tumors reproducibly in the whisker barrel cortex. Fluorescent labeling and cytochrome oxidase staining permit visualization of tumor growth patterns, which disrupt the barrel cortex by microscopic invasion and by gross tissue deformation. Magnetic resonance imaging demonstrates the anatomical extension of these tumors in live rodents. Using this model for further studies on the effects of malignant glioma growth on functional cerebral cortex should advance our understanding of the neurological issues and management of patients with these tumors.

Key Words * C6 glioma * cytoarchitecture * DBT glioma * magnetic resonance imaging * somatosensory cortex * rodent model

Gliomas are the most common supratentorial primary neoplasm of the adult brain.[27] Complete surgical resection of these lesions is difficult, if not impossible, because the tumor cells are found in histologically normal areas of brain that are distant from the bulk tumor.[5,14,21,22] Invasive cells are responsible for tumor recurrence following resection[17] and, through unknown mechanisms, invasive cells cause 
significant neurological dysfunction, even in the absence of a significant mass or recurrence of bulk disease.[23] There is often substantial "silent" tumor growth in the cortex without significant clinical symptoms and signs; cortical areas grossly involved with tumor appear to retain normal function.[19,25] The involvement of cortex subserving known functions, which is present by the time these neoplasms are manifest, limits or even prevents surgical treatment, which may adversely affect patient outcome.[1]

To investigate the biology of these invasive neoplasms in relation to the structure and, ultimately, the function of a well-defined cortical area, a model was developed that involved the use of the rodent whisker barrel cortex.[29,31] The whisker barrels coincide with the primary somatosensory cortex that represents the face in many rodents.[33] A barrel in layer IV is a group of cells related to the corresponding whisker on the opposite face. The barrel cortex was originally recognized as whisker-activated cortex,[30] and later visualized as a whiskerlike array when the brain is cut into sections tangential to the surface of the brain.[31] The unique and highly specific cytoarchitecture makes this an ideal model for a wide range of experimental studies. This cortex and the trigeminal pathway leading to it has been used extensively as a neurodevelopmental, functional, and behavioral model of normal brain. It has also been exploited as a model for evaluating the control of cerebral blood flow and pathological entities such as cerebral ischemia. $[9,13,28]$ The stereotypical anatomy of this cortical region (Fig. 1) enables precise determination of the pathological derangements caused by tumor growth and invasion. The barrel cortex has been well studied. In particular, functional responses to whisker stimulation can be rapidly evaluated with optical imaging and functional magnetic resonance (MR) imaging. $[9,13,36]$ Established rat and mouse models of malignant glioma are available. We targeted these cells to study the effects of tumor growth on the barrel cortex and are unaware of any previous description of the whisker barrel cortex in the context of neoplasia or any other animal models of gliomas in defined functional regions of the brain.

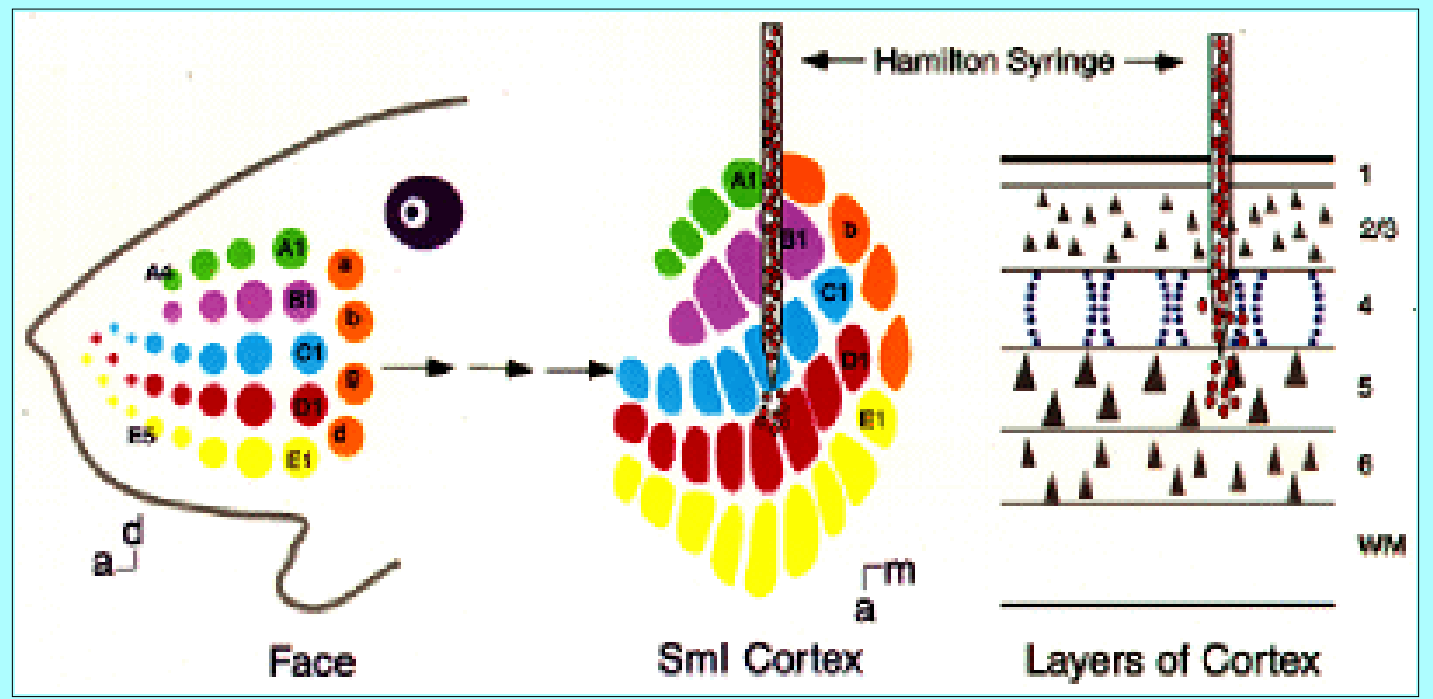

Fig. 1. Schematic drawing showing the projection of the gridlike pattern of whiskers on the rodent face to the gridlike pattern of barrels in layer IV of the contralateral somatosensory cortex. Each whisker and its homologous barrel are uniquely identified by a letter-number code, A1, E5, and so forth. Dil-labeled glioma cells were injected into the center of the barrel field through a small craniostomy targeted by surface landmarks. $a=$ anterior; $d=$ dorsal; $\mathrm{m}=$ medial; $\mathrm{SmI}=$ somatosensory cortex; $\mathrm{WM}=$ white matter. 


\section{Cell Lines}

Rat C6 Cells. The C6 rat glioma cell line was originally isolated from an intracranial tumor in a rat exposed to N-nitroso-methyl-urea and is a reliable cell line model for glioblastoma.[2-4,6,7] For this experiment, C6 cells frozen in culture medium with 5\% dimethylsulfoxide were thawed, washed with magnesium-free Hank's balanced salt solution (HBSS), and grown in Dulbecco's modified Eagle's medium (DMEM) with $15 \%$ heat-inactivated fetal calf serum, $0.2 \mathrm{mM}$ glutamine, $50 \mathrm{mg} / \mathrm{ml}$ neomycin, $100 \mathrm{mg} / \mathrm{ml}$ penicillin, and $100 \mathrm{mg} / \mathrm{ml}$ streptomycin. The cells were then plated in T-75 culture flasks for incubation in a $5 \% \mathrm{CO}_{2}$ humidified atmosphere at $37^{\circ} \mathrm{C}$ and used at low passages.

Mouse DBT Cells. The DBT cell line was originally generated from an intracranial tumor induced in an adult mouse after intracerebral injection of a Rous sarcoma virus.[15] These cells were grown in T-75 culture flasks in the same manner described for the C6 cells.

In vitro Fluorescent Labeling of Tumor Cells. The feeding media of cells in culture at 60 to $80 \%$ confluence (exponential growth phase) was aspirated and the cells were rinsed with HBSS. The lipophilic fluorescent dye Dil was dissolved in $100 \%$ ethanol to a stock concentration of $10 \mathrm{mg} / \mathrm{ml}$. Cell labeling was accomplished by bathing the cells with $0.4 \mathrm{ml}$ stock Dil solution with $9.6 \mathrm{ml}$ feeding medium in the T-75 flasks. Forty-eight hours later, immediately prior to injection, the mixture of Dil and feeding media was aspirated and the cells were washed with HBSS. Standard feeding medium was placed in the flask and the cells were examined using a fluorescence microscope with a rhodamine filter to evaluate labeling.

Preparation of Cells for Injection. Feeding medium was aspirated from flasks of labeled cells and $3 \mathrm{ml}$ of $0.5 \%$ trypsin-ethylenediaminetetraacetic acid was added for 1 minute. When the cells were suspended, the trypsin was inactivated by adding $7 \mathrm{ml}$ DMEM with fetal calf serum. The suspended cells were centrifuged at $250 \mathrm{G}$ for 10 minutes at $4^{\circ} \mathrm{C}$, and the cells were resuspended in $2 \mathrm{ml}$ DMEM. An aliquot of cells was aspirated, stained with trypan blue dye, and counted with a hemocytometer. The cells were once again centrifuged and resuspended in serum-free DMEM to a concentration of 200,000 C6 cells/ $\mu$ l or 150,000 DBT cells/ $\mu$ l and cooled on ice for immediate injection.

\section{Animal Preparation}

Induction of Anesthesia. Adult male Wistar rats weighing $250 \mathrm{~g}$ and adult male BALB/c mice weighing $25 \mathrm{~g}$ were used in the experiments. All animal protocols were approved by the Division of Comparative Medicine and meet or exceed Association for Assessment and Accreditation of Laboratory Animal Care International and National Institutes of Health standards. The mice were anesthetized with $25 \mathrm{mg} / \mathrm{kg}$ ketamine, $5 \mathrm{mg} / \mathrm{kg}$ xylazine, and $2.5 \mathrm{mg} / \mathrm{kg}$ acepromazine via intraperitoneal injection. The rats were anesthetized using a mixture of $10 \mathrm{ml}$ ketamine $(100 \mathrm{mg} / \mathrm{ml})$ and $1.5 \mathrm{ml}$ xylazine $(100 \mathrm{mg} / \mathrm{ml})$ and injected with $0.1 \mathrm{ml} / 100 \mathrm{~g}$ intraperitoneally. All operations were performed on the rodent's left side with magnification provided by either loupes or a wall-mounted microscope. Tumor cells were injected into groups of five animals on the same day.

Rat Surgery. After induction of general anesthesia, a midline scalp incision was made and the periosteum was pulled down sharply; the coronal and lambdoidal sutures were identified. The temporal muscle was freed sharply from the frontal crest (analogous to the superior temporal line in humans) and retracted with No. 4-0 silk suture. A high-speed drill with a 2-mm cutting burr was used to make a craniostomy at the frontal crest 1 to $2 \mathrm{~mm}$ anterior to the midpoint between the coronal and lambdoid 
sutures (cranial landmark for the barrel field). A 10- $\mu$ l Hamilton syringe with $5 \mu$ l of cells was lowered into the cortex with a micromanipulator, and $10^{6} \mathrm{C} 6$ cells in suspension were injected during 1 - to 2 -minute period. The needle was removed, the wound was irrigated with saline, and the craniostomy site was sealed with super glue. The wound was irrigated again, and the scalp closed with No. 4-0 silk sutures.

Mouse Surgery. After incising the scalp in the midline, the periosteum was pulled down sharply. The barrel field is located in the distribution of the middle cerebral artery (MCA) territory in the parietal cortex and is drained by large cortical veins that can be easily seen through the skull. The barrel field itself is devoid of these large cortical veins and it is in this area devoid of veins along the MCA that the barrel field is reliably localized.[8] Because the mouse skull is translucent, the location of the whisker barrel cortex was identified using these vascular landmarks. A 1-mm cutting burr was used to make a craniotomy over the center of the barrel field and a 5- $\mu$ l Hamilton syringe with $3 \mu \mathrm{l}$ of suspended DBT cells was lowered through the craniostomy into the cortex by using a micromanipulator; $4.5 \mathrm{X} 10^{5}$ tumor cells were injected. The craniostomy and scalp were closed as described earlier for the rats.

\section{Magnetic Resonance Imaging Studies}

Rat Studies. Proton $\mathrm{T}_{2}$-weighted MR images were collected using fast spin echo with an effective echo time (TE) of $80 \mathrm{msec}$. The images were acquired using the following parameters: 5 -second repetition time (TR), 20-msec echo spacing time, 256 X 256 data matrix size (resulting in a plane resolution of 117 $\mu \mathrm{m}$ ), 1-mm slice thickness, and $3 \times 3 \mathrm{~cm}^{2}$ field of view (FOV). The rats were anesthetized with halothane/oxygen prior to the acquisition of MR images. All images were acquired using a 3-cm internal diameter quadrature radiofrequency coil. Body temperatures were maintained at $37^{\circ} \mathrm{C}$ by using a heating pad.

Mouse Studies. Proton $\mathrm{T}_{2}$-weighted and diffusion-weighted spin-echo MR images were acquired using a 1.5-cm outer diameter circular surface receiver coil and a 12-cm inner diameter Helmholtz transmit coil. The $\mathrm{T}_{2}$-weighted images were acquired using a 3-second TR, 50-msec TE, $128 \mathrm{X} 128$ data matrix (resulting in a plane resolution of $117 \mu \mathrm{m}$ ), $0.5-\mathrm{mm}$ slice thickness, and $1.5 \mathrm{X} 1.5-\mathrm{cm}^{2} \mathrm{FOV}$. The diffusion-weighted images were acquired using a 1.5-second TR, 42-msec TE, $128 \mathrm{X} 128$ data matrix (117- $\mu \mathrm{m}$ plane resolution), 1-mm slice thickness, and $1.5 \times 1.5-\mathrm{cm}^{2}$ FOV. The mice were anesthetized with a mixture of halothane and oxygen prior to the acquisition of images and their body temperatures were maintained at $37^{\circ} \mathrm{C}$ by using a heating pad.

\section{Tissue Preparation}

Some animals became symptomatic (lethargic or hemiparetic) 6 to 14 days after tumor cell injection. Symptomatic and asymptomatic animals in a group were given an overdose of anesthetic agent and perfused transcardially with heparinized saline followed by buffered $4 \%$ paraformaldehyde. The rodent brains were removed and placed in $4 \%$ paraformaldehyde with $30 \%$ sucrose for 24 to 48 hours at $4{ }^{\circ} \mathrm{C}$ until the specimens sunk. Whole-brain specimens were photographed using direct and fluorescent illumination. The cerebral cortex was dissected from the thalamus, following which it was flattened, frozen, and cut serially with a vibratome into $50-\mu \mathrm{m}$ sections tangential to the pia mater in a plane parallel to the cortical layers.[26] The sections were collected in phosphate buffer, mounted on slides with mounting medium, coverslipped, sealed, and examined, and photographed using fluorescence and dark-field microscopy. After photography, the cover slips were removed and the sections were floated in 
phosphate buffer and prepared for staining with cytochrome oxidase.

\section{Cytochrome Oxidase Staining}

Histochemical investigation for the mitochondrial enzyme cytochrome oxidase provides excellent visualization of the whisker barrels.[34,35] Briefly, the tissue sections were incubated in a solution of cytochrome C (22 mg), diaminobenzidine tetrachloride $(50 \mathrm{mg})$, and sucrose $(4 \mathrm{~g})$ in $45 \mathrm{ml}$ distilled water $/ 45 \mathrm{ml} 0.2 \mathrm{M}$ sodium phosphate buffer at $37^{\circ} \mathrm{C}$ for 2 to 4 hours in the dark. The sections were rinsed three times in $0.1 \mathrm{M}$ phosphate buffer, mounted on slides, and allowed to dry. The slides were cleared in xylene for 1 hour and coverslipped with DPX.

\section{Camera Lucida Techniques}

Sections stained with cytochrome oxidase were drawn through a light microscope equipped with a camera lucida drawing tube. Serial sections of the barrel field and tumor were aligned and traced with perforating vessels as fiducial markers. Drawings were scanned into a computer equipped with photographic and drawing software.

\section{Anatomical Correlation of Images}

Complete MR imaging series through the brain from the occipital pole to the frontal pole were collected at slice thicknesses of $1 \mathrm{~mm}$ in the rat and $0.5 \mathrm{~mm}$ in the mouse. From these, the level of each coronal MR imaging slice was determined on the gross brains. Landmarks (thalamus, hippocampus, ventricles, and so forth) were used to made a reasonable estimate as to the location of the barrel field on the MR images by comparing them to coronal sections in standard rodent brain atlases. Finally, the location of the MR imaging coronal slices relative to the barrel field reconstructions were determined using the position of the MCA relative to the barrel fields and as projected on the gross specimens.

\section{Sources of Supplies and Equipment}

The C6 rat glioma cells were obtained from American Type Tissue Collection (Rockville, MD) and the Wistar rats and BALB/c mice from Charles River Laboratories (Wilmington, MA). The dimethylsulfoxide was purchased from Sigma Chemical Co. (St. Louis, MO) and the HBBS, DMEM, trypsin-ethylenediaminetetraacetic acid, and trypan blue dye from Gibco BRL (Grand Island, NY). Fetal calf serum was obtained from Gemini Bioproducts (Calabasa, CA).

Dil was purchased from Molecular Probes (Eugene, OR). The rhodamine filter that was used with the fluorescence microscope was purchased from Olympus (Lake Success, NY) and the wall-mounted microscope and light microscope with camera lucida drawing tube from Carl Zeiss Inc. (Thornwood, $\mathrm{NY})$.

Dremel (Racine, WI) manufactured the high-speed drill used in surgery. The Vectashield mounting medium was obtained from Vector Laboratories (Burlington, ON, Canada).

Adobe Photoshop (version 4.01) and Illustrator (version 7.0) were obtained from Adobe Systems, Inc. (Mountain View, CA).

\section{RESULTS}

\section{In vitro Labeling of Glioma Cell Lines}


The cells were successfully labeled with Dil, a lipophilic fluorescent marker, without affecting cell viability in culture. Cell counts obtained using a hemocytometer indicated 95 to $98 \%$ viability in both the C6 and DBT cell populations 48 hours after staining with Dil. All cells in the T-75 flasks were labeled with Dil as confirmed just prior to cell injection (Fig. 2).

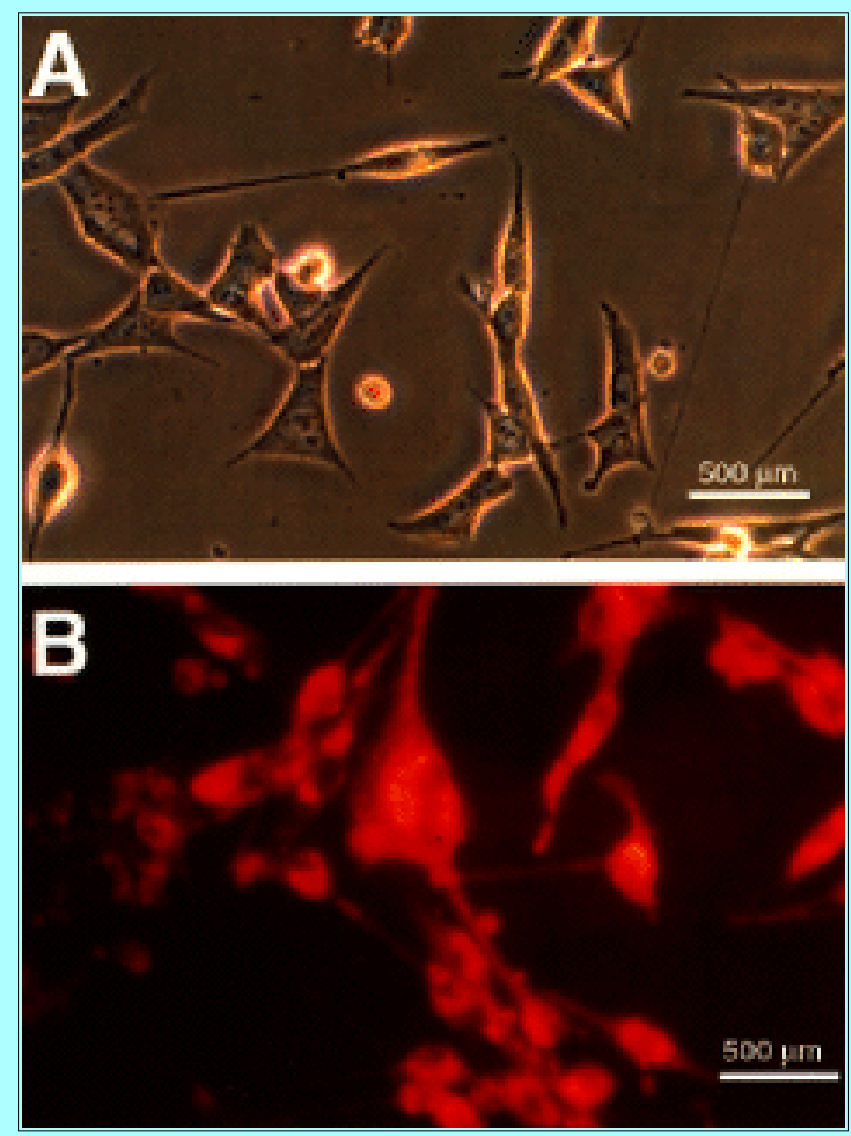

Fig. 2. Phase-contrast (A) and fluorescence (B) photomicrographs of DBT mouse glioma cells in culture labeled with Dil before injection. All cells were labeled with Dil without affecting cell viability. Original magnification X 20.

\section{In vivo Tumor Development}

Once the procedures were established, all 20 rats $(100 \%)$ and 10 mice (100\%) developed tumors following injection of $10^{6}$ cultured C6 cells and $4.5 \times 10^{5}$ DBT cells, respectively. All 15 symptomatic animals showing lethargy and hemiparesis had large tumors on the left cerebral hemisphere at the site of injection. Five animals developed extraaxial intracranial masses, which were likely the result of subarachnoid injection of tumor cells or direct extension of tumor from the cortex. Fifteen animals with tumors were asymptomatic at the time of death; however, all four symptomatic animals that were not killed within 24 hours progressed rapidly to death. All tumors were Dil positive based on histological analysis.

\section{Magnetic Resonance Imaging}

Coronal $\mathrm{T}_{2}$-weighted $\mathrm{MR}$ images revealed abnormal signal changes in all five rats that underwent imaging. Hyperintense intraaxial masses in the left parietal region were typically found at the injection site. Magnetic resonance images usually were performed once the animal became symptomatic; however, this was associated with a high anesthesia-related rate of mortality (30\% of symptomatic animals). One 
asymptomatic animal underwent imaging on postinjection Day 5 and a mass was present (Fig. 3A). The following day, the animal became lethargic and hemiparetic and repeated MR imaging revealed significant tumor enlargement (Fig. 3B).

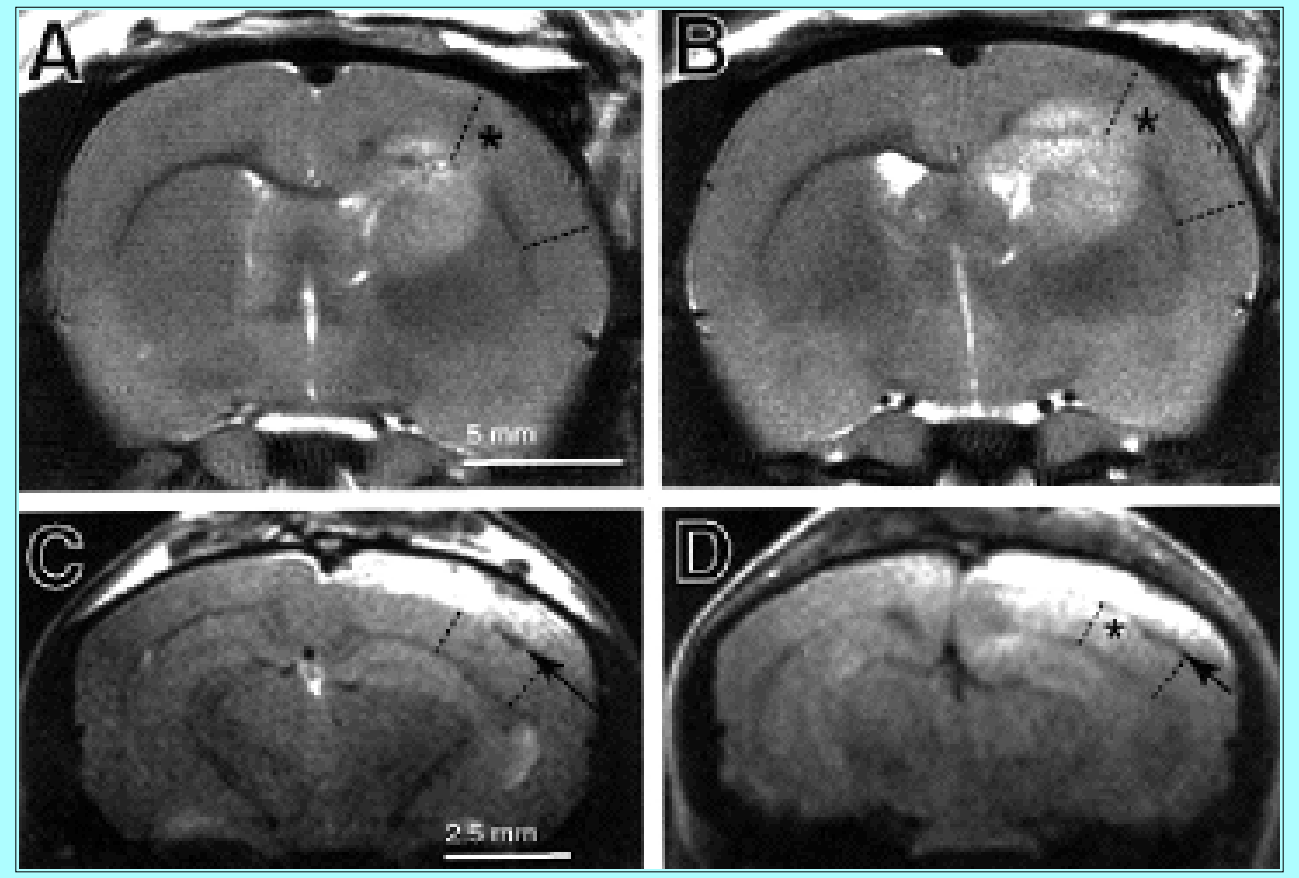

Fig. 3. Magnetic resonance images of rodent brains with experimental gliomas in vivo. A and B: Intraaxial tumor in a rat 5 and 6 days, respectively, after injection of $10^{6} \mathrm{C} 6$ cells demonstrating the rapid growth of this tumor. This animal had no overt impairments when it first underwent imaging (A), but had developed contralateral hemiparesis by the time of the second imaging session (B). The area of the barrel field is indicated by hatching as well as the tumor within the cerebral cortex (asterisks). $\mathrm{C}$ : $\mathrm{A} \mathrm{T}_{2}$-weighted image of mouse brain demonstrating significant extra- and intraaxial tumor (arrow marks the interface) that depresses the cortex and produces a midline shift 8 days after injection of $4 \times 10^{5} \mathrm{DBT}$ cells. This animal showed no overt impairment. D: Diffusion-weighted image obtained in the same mouse, revealing a signal change in the cortex underlying the bulk tumor, suggesting cortical invasion of tumor (asterisk), which was confirmed by histological analysis. The area of the barrel field is indicated by hatching. Both brains are also shown in Fig. 4 and in the reconstructed barrel fields in Fig. 7 for comparison.

Coronal $\mathrm{T}_{2}$-weighted and diffusion-weighted $\mathrm{MR}$ images obtained in five mice that were implanted with DBT cells also demonstrated intracranial neoplasms. The masses were hyperintense. The example shown in Fig. 3C illustrates a large extraaxial component that was probably the result of subarachnoid tumor injection or tumor extended from the cortex into the subarachnoid space. Intraparenchymal signal changes seen on diffusion-weighted images were consistent with tumor involvement of the cerebral cortex (Fig. 3D).

All animals were perfused shortly after the MR images had been obtained to evaluate the anatomical correlation between the MR images and the perfused specimen. All rats and mice in which there was MR imaging evidence of tumor had Dil-positive gross tumors and tissue in the region in which signal change abnormalities were observed on MR imaging. 


\section{Fluorescence Microscopy}

After they were perfused and removed, the brains were photographed using both incidental and fluorescent illumination. Left cerebral hemispheres were enlarged, compared with right hemispheres, in animals with intraaxial masses (Fig. 4A) and the left hemisphere was deformed and compressed in those animals with extraaxial tumor involvement (Fig. 4B). Grossly, all tumors were Dil positive.

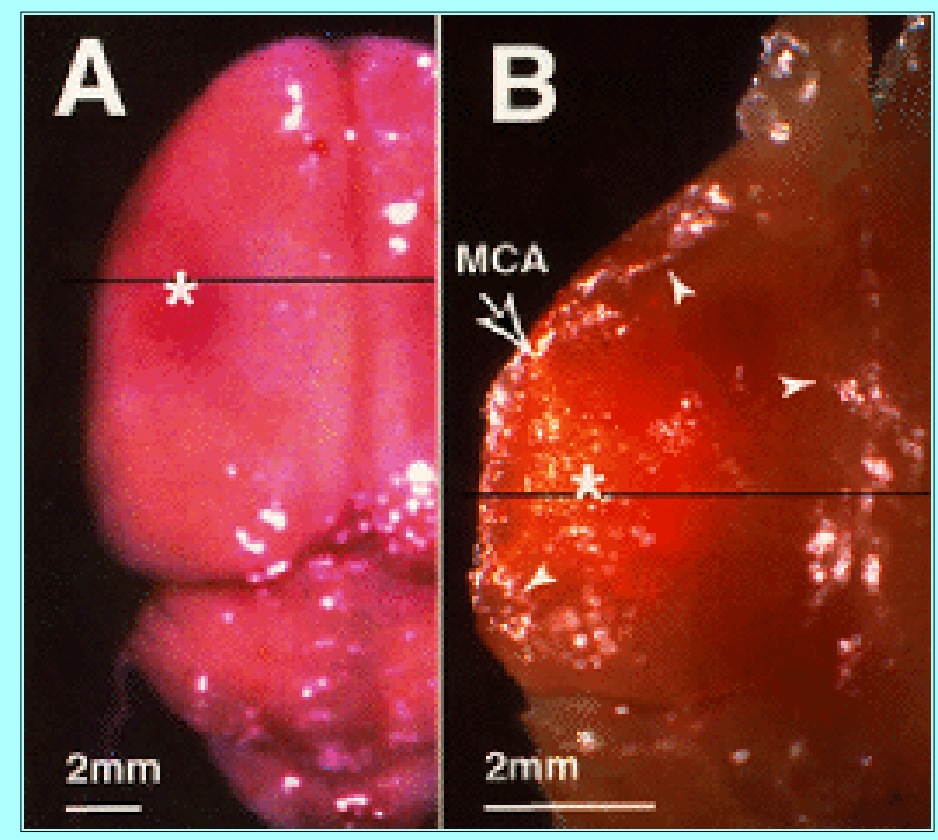

Fig. 4. Photographs showing dorsal views of the left side of fixed rat (A) and mouse (B) brains into which C6 and DBT cells, respectively, were injected. Dil-labeled tumors (asterisks) are red with incidental (A) and bright orange with fluorescent (B) illumination. The edges of the concave cortical impression from the extraaxial mass in the mouse are indicated (arrowheads). The DBT tumor cells in the mouse brain follow the principal branches of the MCA (arrow). The MR images of these animals are shown in Fig. 3 and the level at which the MR image was obtained is indicated by the solid lines in the coronal plane. Anterior is above the line.

Histological sections of brain were examined using fluorescence microscopy. All areas of bulk tumor exhibited positivity for Dil. Some areas of bulk tumor were less fluorescent than other areas; this was related to Dil dilution through mitosis. Dil-positive cells distant from the bulk tumor were seen in the injected hemisphere in all cases. In both C6 and DBT cells, the invasion of tumor cells away from the bulk tumor grew along arterial but not venous trees (Fig. 5). Generally, invasion away from the bulk tumor was greater in the C6 model than the DBT model, which may reflect inherent differences between the cell lines. 


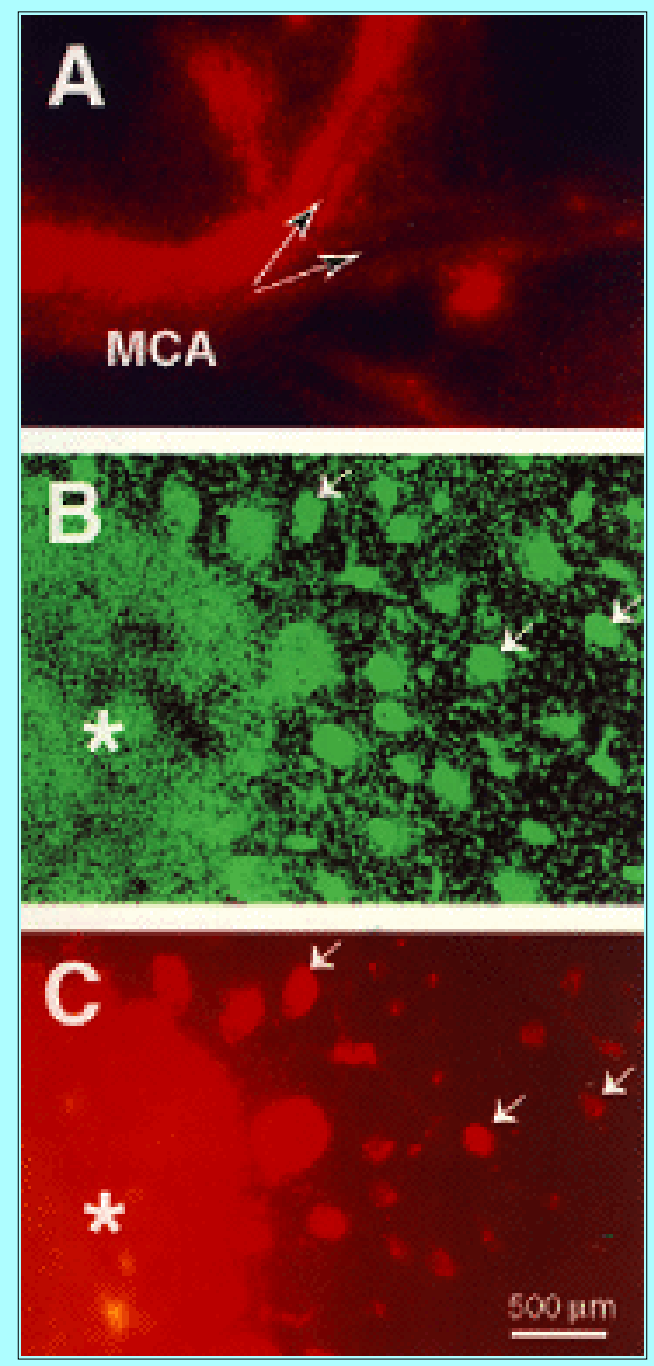

Fig. 5. Fluorescence photomicrographs of tangential sections of rat brain, obtained 6 days after injection of C6 cells, showing the relationship of fluorescent tumor cells to surface vessels and the patterns of intraparenchymal spread. Both C6 and DBT (not shown) tumor cells follow arterial, but not venous, vessels on the brain surface and within the brain parenchyma. A: Surface section demonstrating spread of tumor cells (arrows) along the MCA. B: Tangential section stained with the nuclear marker bisbenzamide demonstrating tumor cell viability within the bulk tumor (asterisk) and invasion along penetrating arterioles (arrows) as visualized using an ultraviolet filter. C: Same section as that shown in B visualized with a rhodamine filter showing Dil-positive tumor spreading along intraparenchymal arterioles (arrows). The area of bulk tumor is indicated (asterisk). Dil-labeled perivascular tumor cell locations coincide with most of the cell groups stained in $\mathrm{B}$, but the extent of Dil labeling is restricted, which is consistent with Dil dilution in proliferation.

\section{Cytochrome Oxidase Histological Findings}

Cytochrome oxidase staining was performed to visualize the whisker barrel cortex in layer IV of the somatosensory cortex in both injected and noninjected (control) hemispheres to evaluate tumor location and tumor disruption and/or deformation of the barrel cortex. All tumors grossly involved the whisker barrel cortex. In areas of bulk tumor, the barrel field was invaded and its architecture modified (Fig. 6). 


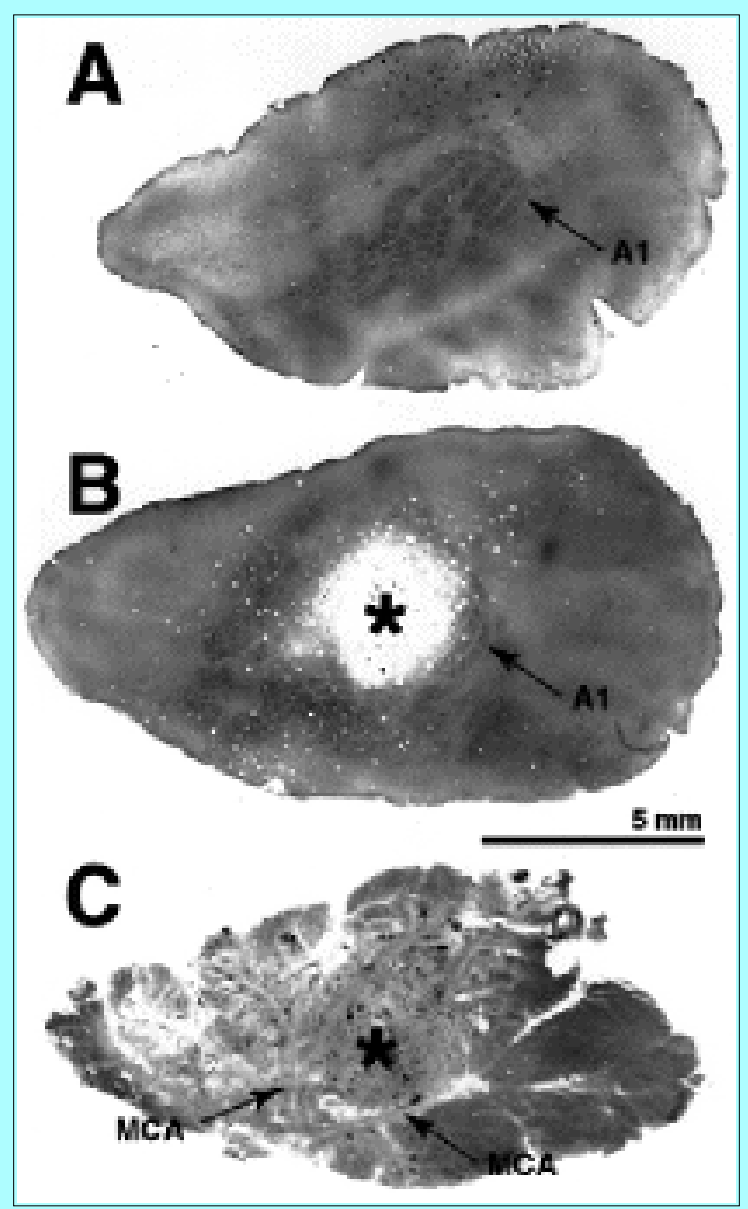

Fig. 6. Tangential cytochrome oxidase-stained sections of rat brain obtained 6 days after injection of C6 cells (same specimen as that shown in Figs. 3-5). A: Stained sections form the contralateral "control" (noninjected) barrel field from the same rat. The image is in the same orientation as the sections from the tumor-injected side for comparison. The A1 barrel is identified (see Fig. 1 for nomenclature). B: Similar section from the injected hemisphere demonstrating the disruption of the stained barrel pattern by intraaxial tumor (asterisk). The A1 barrel is displaced by tumor relative to other barrels (see Fig. 7). C: Tangential section from cortical surface. Tumor (asterisk) surrounds the MCA.

In areas adjacent to bulk tumor without gross invasion of the barrels, there was also distortion of the normal barrel pattern. A comparison of cytochrome oxidase staining patterns of injected and noninjected hemispheres, as well as barrel field reconstruction using camera lucida techniques (Fig. 7), revealed the extent to which the tumor distorted the organization of the somatosensory cortex. This distortion of the barrel pattern may be due to a mass effect from the bulk tumor, infiltration of adjacent barrels by tumor, or both. 


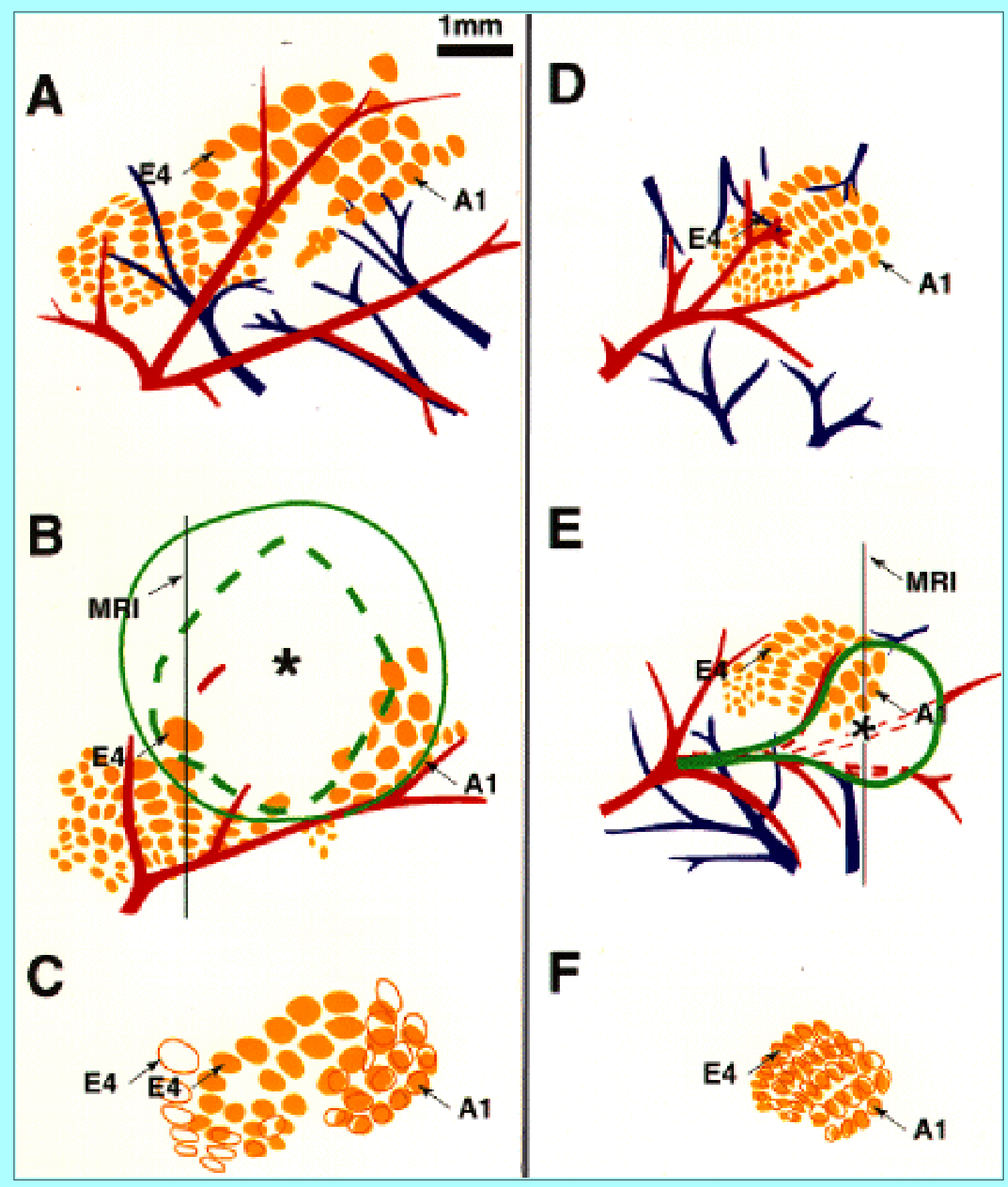

Fig. 7. Camera lucida drawings made from serial sections demonstrating the full barrel field in the rat (A-C) and the mouse (D-F) 6 and 8 days after implantation of C6 and DBT cells, respectively. Comparison to the barrel field patterns on the uninjected "control" sides (A and D) demonstrates the extent of infiltration into and displacement of the somatotopic barrel pattern (B and E). A: Contralateral (normal) barrel field in the rat. B: Tumor in the barrel field (solid green line outlines the tumor at the surface of the brain; dashed green line outlines the tumor in layer IV) in the rat barrel field with invasion and displacement of the barrels (same rat specimen as seen in Figs. 3-5). C: Superimposition of the noninjected barrel field on the injected barrel field of the rat reveals significant destruction and displacement of the barrel field by a malignant C6 glioma. D: Contralateral noninjected (normal) mouse barrel field. E: Tumor (outlined by green) over the barrel field of the mouse with distortion of the barrel architecture (same mouse as in that seen in Figs. 3C and D and 4B). F: Superimposition of the injected and noninjected barrel fields of a mouse with a DBT glioma at the cortical surface demonstrating subtle distortion of the barrel pattern with 
swelling of the barrels, which is probably the result of tumor invasion.

\section{DISCUSSION}

\section{Whisker-Barrel Cortex}

The large whiskers (vibrissae) on the faces of rats and mice are arranged in rows (Fig. 1). In layer IV of the primary somatosensory cortex of these rodents, the layout of this cortical representation is seen in whole or can be reconstructed from serial sections tangential to the pia mater. The cortical barrel field is thus a visible rodent equivalent of the "homunculus," in which each barrel corresponds to a specific whisker (Figs. 1 and 7). Other barrels are related to the smaller hairs around the mouth, on the eyebrows, on the cheek, and at the wrist, and to glabrous pads of the feet. This stereotypical anatomical pattern varies little from animal to animal, permitting comparisons between the normal pattern and changes caused by experimental manipulations as described here. Changes can be measured and evaluated.[32] The detailed function of whisker barrel cortex is also well understood. Two methods that have been used clinically that also have been demonstrated in rodents are of particular interest here: the so-called intrinsic optical signal and functional MR imaging. Both correlate well with barrels during whisker stimulation. $[9,13,36]$

\section{Effects of Neoplasia on Neurological Function}

In the setting of neoplasia, the rodent barrel cortex offers potential insight into the mechanisms through which gliomas impair neurological dysfunction. Neurological dysfunction secondary to gliomas is variable: some patients with large tumors have only minimal symptoms despite extensive involvement of sensory, motor, or other "eloquent" cortex. Intracranial gliomas may reach very large sizes before becoming symptomatic; at which time patients, if left untreated, suffer a rapid decline and subsequent death.[11,18] This glioma-barrel cortex model appears similar: once the animals became symptomatic, if they were not killed, death occurred in most by 12 hours and in all by 24 hours. Before becoming symptomatic, all animals appeared normal, despite an obviously large tumor volume demonstrated in the barrel cortex.

The exact mechanisms for neurological dysfunction resulting from intracranial neoplasia are unknown. Although probably multifactorial, disruption of neuronal function secondary to brain invasion, mass effect with compression of neural structures, and subsequent cerebral edema of the brain adjacent to tumor, resulting in neuronal dysfunction, are clear hypotheses. These proposals, however, are inadequate because a functional area of brain that is grossly involved with tumor may retain its function when tested intraoperatively.[19,25] Evidence suggests that even more complex mechanisms, such as the link between local cerebral blood flow and neuronal activation in the region of neoplasia, may be altered or deranged in such a way as to result in neuronal dysfunction.[20] The present model was developed to elucidate these mechanisms because the function of the whisker barrel cortex can be studied physiologically while its distinct architecture provides an ideal context for precise coupling of neuronal dysfunction and histopathology.

\section{The C6 and DBT Cell Lines as Models for Glioblastoma Multiforme}

Numerous experiments in which the C6 rat glioma implantation model has been used have demonstrated characteristics consistent with human glioblastoma multiforme. The C6 cells invade the rat brain after implantation and single cells have been detected in the contralateral hemisphere. After implantation, C6 
cells also generate bulk tumors, stain positively for glial fibrillary acidic protein, and demonstrate histopathological features of glioblastoma.[2-4,6,7] In the C6 rat implantation model used in this study, invasion away from the bulk tumor grew along arterial, but not venous, trees. This has been seen in other experiments in which C6 gliomas and human gliomas implanted in the rat grew along the vascular basement membrane of arteries, but did not disrupt the vascular lumen.[4,12] Also, studies have shown that early brain invasion occurs in the region of brain adjacent to the vascular basement membrane.[16] The constituents of the vascular basement membrane, especially collagen type IV, coupled with differences in the extracellular matrix in different structures, may provide the substrate necessary for tumor growth and invasion.[12]

The DBT cell line was developed from an intracranial tumor generated by an intracerebral injection of a Rous sarcoma virus.[15] Unpublished data obtained by one of the authors (M.R.C.) indicate that DBT cells have a structure similar to C6 cells grown in culture, are motile and glial fibrillary acidic protein positive, and develop multiple multicellular colonies suspended in gelatinous agar. Injecting these cells into mice, as we did here, also resulted in tumor formation with invasion of cells into the regions beyond bulk tumor.

\section{The Malignant Glioma-Whisker Barrel Model}

After preliminary trials to perfect surgical technique, all animals injected with C6 and DBT cell lines developed intracranial tumors in the region of the whisker barrel cortex. Localization of the barrel cortex is reliable when using the techniques described earlier, because the barrel field was involved with neoplasm in all cases. Gross localization of the barrel field in this way (that is, craniostomy rather than craniotomy) permits reoperation for optical imaging with minimal scarring.

The structure of the barrel field allows for precise evaluation of histopathological conditions induced by malignant gliomas. The barrel field also allows for physiological study of cortical activity through optical imaging techniques. $[9,13]$ In fact, precise localization of a single barrel through stimulation of a single whisker has been demonstrated, as well as quantitative changes in the diameter of pial vessels in response to whisker stimulation.[10] Also, single-unit electrical recording from the somatosensory barrel cortex can be used to evaluate the electrophysiological properties of cortical function in this region.[24] Subsequent studies using this model coupled with the aforementioned techniques may yield qualitative and quantitative physiological data of functional cerebral cortex and its response to malignant gliomas.

Examination of rodents by using MR imaging provides numerous opportunities to develop our understanding of these malignant glial neoplasm. This study shows that these neoplasms can be followed with serial MR imaging, which allows for quantitative growth estimates in vivo. However, volumetric studies in which MR imaging signal abnormality volume and histological tumor volume are compared need to be performed. Studies of patients have shown that areas of $\mathrm{T}_{2}$ signal abnormality distant from bulk tumor have histopathological evidence of neoplastic involvement.[14] In the current study, $\mathrm{T}_{2}$-weighted and diffusion-weighted MR images demonstrated signal abnormality in the region of the barrel cortex and corresponded well with the anatomical location of the tumors based on the histological sections; however, the signal change was more extensive than of bulk tumor found histologically. Further studies are necessary in which MR imaging is used to establish volumetric data as well as studies utilizing functional MR imaging to help evaluate cortical function in the presence of invasive gliomas.

Magnetic resonance imaging also offers the potential to assess responses to therapeutic measures such as 
surgery, radiation and chemotherapy. Conversely, changes in cortical function may also be assessed following therapy using this model.

\section{CONCLUSIONS}

These studies demonstrate that the DBT and C6 glioblastoma cell lines reproducibly generate malignant glial tumors in the whisker barrel cortex of mice and rats, respectively. Fluorescent labeling permits precise visualization of the anatomical patterns of growth and invasion of these tumors in relation to the ordinarily highly ordered cytoarchitecture of the rodent barrel cortex. Tumor growth disrupts the barrel cortex by microscopic invasion and by gross tissue deformation.

Magnetic resonance imaging demonstrated the anatomical extent of DBT and C6 rodent gliomas in live animals. The growth of these tumors was followed sequentially with MR images obtained at daily intervals. Further evaluation of the effects of malignant glioma growth on functional cerebral cortex based on this model could improve our understanding and management of patients who experience neurological deficits from these tumors.

\section{Acknowledgments}

The authors are indebted to Michael Lai, Ph.D., of the Department of Microbiology at the University of Southern California for generously providing the DBT cell line. We are also grateful to Brad Miller, $\mathrm{Ph}$.D., for his expertise and assistance with microscopy and photography, to Bertha McClure and Jon Christensen for the histological studies, to Michael Zahner for the tissue culture, and to Kathy Diekmann for preparation of this manuscript. The authors especially wish to thank Jeffrey Ojemann, M.D., whose insight into cortical activation and tumor biology suggested the development of this model.

\section{References}

1. Ammirati M, Vick N, Liao Y, et al: Effect of the extent of surgical resection on survival and quality of life in patients with supratentorial glioblastomas and anaplastic astrocytomas. Neurosurgery 21:201-206, 1987

2. Bernstein JJ, Goldberg WJ, Laws ER Jr, et al: C6 glioma cell invasion and migration of rat brain after neural homografting: ultrastructure. Neurosurgery 26:622-628, 1990

3. Bernstein JJ, Laws ER Jr, Levine KV, et al: C6 glioma-astrocytoma cell and fetal astrocyte migration into artificial basement membrane: a permissive substrate for neural tumors but not fetal astrocytes. Neurosurgery 28:652-658, 1991

4. Bernstein JJ, Woodard CA: Glioblastoma cells do not intravasate into blood vessels. Neurosurgery 36:124-132,1995

5. Burger PC, Dubois PJ, Schold SC, et al: Computerized tomographic and pathologic studies of the untreated, quiescent, and recurrent glioblastoma multiforme. J Neurosurg 58:159-169, 1983

6. Chicoine MR, Silbergeld DL: Assessment of brain tumor cell motility in vivo and in vitro. J Neurosurg 82:615-622, 1995

7. Chicoine MR, Silbergeld DL: Invading C6 glioma cells maintaining tumorigenicity. J Neurosurg 83:665-671, 1995 
8. Cox SB, Woolsey TA, Rovainen CM: Localized dynamic changes in cortical blood flow with whisker stimulation corresponds to matched vascular and neuronal architecture of rat barrels. J Cereb Blood Flow Metab 13:899-913, 1993

9. Dowling JL, Henegar MM, Liu D, et al: Rapid optical imaging of whisker responses in the rat barrel cortex. J Neurosci Methods 66:113-122, 1996

10. Erinjeri JP, Liu D, Woolsey TA: Timing of intrinsic optical and pial vessel diameter changes during activation of mouse barrel cortex. Soc Neurosci Abstr 42:632, 1998 (Abstract)

11. Frankel SA, German WJ: Glioblastoma multiforme. Review of 219 cases with regard to natural history, pathology, diagnostic methods, and treatment. J Neurosurg 15:489-503, 1958

12. Giese A, Westphal M: Glioma invasion in the central nervous system. Neurosurgery 39:235-252,1996

13. Hodge CJ Jr, Stevens RT, Newman H, et al: Identification of functioning cortex using cortical optical imaging. Neurosurgery 41:1137-1145, 1997

14. Kelly PJ, Daumas-Duport C, Kispert DB, et al: Image-based stereotaxic serial biopsies in untreated intracranial glial neoplasms. J Neurosurg 66:865-874, 1987

15. Komanishi T: Brain tumors induced with Rous sarcoma virus, Schmidt-Ruppin strain. I. Introduction of brain tumors in adult mice with Rous chicken sarcoma cells. Japan J Exp Med 37:461-474, 1967

16. Nagano N, Sasaki H, Aoyagi M, et al: Invasion of experimental rat brain tumor: early morphological changes following microinjection of C6 glioma cells. Acta Neuropathol 86:117-125, 1993

17. Nazzaro JM, Neuwelt EA: The role of surgery in the management of supratentorial intermediate and high-grade astrocytomas in adults. J Neurosurg 73:331-344, 1990

18. Netsky MG, August B, Fowler W: The longevity of patients with glioblastoma multiforme. J Neurosurg 7:261-269, 1950

19. Ojemann JG, Miller JW, Silbergeld DL: Preserved function in brain invaded by tumor.

Neurosurgery 39:253-259, 1996

20. Ojemann JG, Neil JM, MacCleod AM, et al: Increased functional vascular response in the region of a glioma. J Cereb Blood Flow Metab 18:148-153, 1998

21. Scherer HJ: The forms of growth in gliomas and their practical significance. Brain 63:1-35, 1940

22. Silbergeld DL, Chicoine MR: Isolation and characterization of human malignant glioma cells from histologically normal brain. J Neurosurg 86:525-531, 1997

23. Silbergeld DL, Rostomily RC, Alvord EC Jr: The cause of death in patients with glioblastoma is multifactorial: clinical factors and autopsy findings in 117 cases of supratentorial glioblastoma in adults. J Neurooncol 10:179-185, 1991

24. Simons DJ: Neuronal integration in the somatosensory whisker/barrel cortex, in Jones EG, Diamond IT (eds): The Barrel Cortex of Rodents. Cerebral Cortex, Vol 11. New York: Plenum Press, 1995, pp 
25. Skirboll SS, Ojemann GA, Berger MS, et al: Functional cortex and subcortical white matter located within gliomas. Neurosurgery 38:678-685, 1996

26. Strominger RN, Woolsey TA: Templates for localizing the whisker area in fresh flattened mouse and rat cortex. J Neurosci Methods 22:113-118, 1987

27. Tooth HH: Some observations on the growth and survival period of intracranial tumours, based on the records of 500 cases, with special reference to the pathology of the gliomata. Brain 35:61-108, 1912 28. Wei L, Rovainen CM, Woolsey TA: Ministrokes in rat barrel cortex. Stroke 26:1459-1462, 1995 29. Welker C, Woolsey TA: Structure of layer IV in the somatosensory neocortex of the rat: description and comparison with the mouse. J Comp Neurol 158:437-454, 1974

30. Woolsey TA: Somatosensory, auditory and visual cortical areas of the mouse. Johns Hopkins Med J 121:91-112, 1967

31. Woolsey TA, Van der Loos H: The structural organization of layer IV in the somatosensory region (SI) of mouse cerebral cortex. The desription of a cortical field composed of discrete cytoarchitectonic units. Brain Res 17:205-242, 1970

32. Woolsey TA, Wann JR: Areal changes in mouse cortical barrels following vibrissal damage at different postnatal ages. J Comp Neurol 170:53-66, 1976

33. Woolsey TA, Welker C, Schwartz RH: Comparative anatomical studies of the SmI face cortex with special reference to the occurrence of "barrels" in layer IV. J Comp Neurol 164:79-94, 1975

34. Wong-Riley M: Changes in the visual system of monocularly sutured or enucleated cats demonstrable with cytochrome oxidase histochemistry. Brain Res 171:11-28, 1979

35. Wong-Riley MTT, Welt C: Histochemical changes in cytochrome oxidase of cortical barrels after vibrissal removal in neonatal and adult mice. Proc Natl Acad Sci USA 77:2333-2337, 1980

36. Yang X, Hyder F, Shulman RG: Functional MRI BOLD signal coincides with electrical activity in the rat whisker barrels. Magn Reson Med 38:874-877, 1997

Manuscript received March 8, 1999.

Accepted in final form July 9, 1999.

This study was supported in part by National Institutes of Health Grant Nos. P01-NS17663 and R01-NS28781 to Dr. Woolsey and T32-NS07205 to Dr. Sherburn, and by a grant from The Spastic Paralysis Foundation of the Illinois/Eastern Iowa District of the Kiwanis International to Dr. Woolsey.

Address reprint requests to: Thomas A. Woolsey, M.D., Department of Neurosurgery, Washington University School of Medicine, 4566 Scott Avenue, Box 8057, St. Louis, Missouri 63110. email: diekmank@medicine.wustl.edu. 